\title{
Pose-Based 3D Human Motion Analysis Using Extreme Learning Machine
}

\author{
Arif Budiman \\ Computer Science Faculty, University of Indonesia \\ West Java, Indonesia 16424 \\ Email: arif.budiman21@ui.ac.id
}

\author{
Mohamad Ivan Fanany \\ Computer Science Faculty, University of Indonesia \\ West Java, Indonesia 16424 \\ Email: ivan.fanany@cs.ui.ac.id
}

\begin{abstract}
In 3D human motion pose-based analysis, the main problem is how to classify multi-class label activities based on primitive action (pose) inputs efficiently for both accuracy and processing time. Because, pose is not unique and the same pose can be anywhere on different activity classes. In this paper, we evaluate the effectiveness of Extreme Learning Machine (ELM) in 3D human motion analysis based on pose cluster. ELM has reputation as eager classifier with fast training and testing time but the classification result originally has still low testing accuracy even by increasing the hidden nodes number and adding more training data. To achieve better accuracy, we pursue a feature selection method to reduce the dimension of pose cluster training data in time sequence. We propose to use frequency of pose occurrence. This method is similar like bag of words which is a sparse vector of occurrence counts of poses in histogram as features for training data (bag of poses). By using bag of poses as the optimum feature selection, the ELM performance can be improved without adding network complexity (Hidden nodes number and training data).
\end{abstract}

\section{INTRODUCTION}

Nowadays, the most challenging task in computer vision based human motion analysis is to understand and recognize human action in semantic interpretation (high level vision) rather than intermediate-level (human tracking) or low-level vision (Human detection) [12]. Semantic meaning of action recognition is actually a task to label or classify an activity motion as belongs to one of some meaningful action classes by using machine learning algorithms. The classifier should perform efficiently to give both well-acceptance accuracy and fast processing time especially for real time application.

Extreme Learning Machine (ELM) has reputation as "extreme" in processing speed machine learning [3], however, the effectiveness of ELM in human motion analysis is largely unknown. How ELM, as eager classifier with fast processing time reputation, can deal with human activity classification based on pose cluster with semantic meaning. Such problem has reputation as hard classification problem due to pose cluster characteristic. Pose cluster in motion activity has feature characteristic for pose position in time sequence (spatiotemporal) and the frequency of pose occurrence in motion sequence. Both features are not unique and could be anywhere distributed on different activity classes. The distribution of features depends on the regularity of motion activity. For example, dance actions can be viewed as more regular than badminton sport actions. Dance action has a more strict rule followed by the dancer during her performance. In this paper, we evaluate the effectiveness of ELM classifier to deal with Balinese traditional dance and badminton sport. Both have different feature characteristic and it is difficult to find a deterministic function to select the optimal features and optimal classifier structure.

ELM itself has two major issues need to be addressed to improve its accuracy [2], [5] :

1) The structure size of the number of hidden nodes. The optimal number is still unknown with trial-and-error.

2) Whether the computation complexity can be further reduced when given large number of training data and when large number of hidden nodes required.

Our contribution is how the effective learning method of ELM to deal with 3D human motion pose-based classification problem to give not only processing speed but also wellacceptance accuracy by using the efficient network structure.

\section{RELATED WORKS}

The taxonomy of Human actions distinguishes actions into action primitive (called pose), action and activity [10]. Pose is a set of features of body part location and can be interpreted as meaningful string of symbol to describe the activity. Different activities may have more than one similar poses anywhere determined by pose location and the frequency of pose occurrence.

Using Kinect as a motion capture device [7], the human motion was constructed from instance of skeleton features in 3D space. An instance of skeleton features constructs a key body pose. A sequence of key poses forms a basic (primitive) motion. Clustering of data observation in feature space is the most common approach to identify key poses and then classify it using common classifier such as nearest neighbors or support vector machine. Another hot issue classifier nowadays is Extreme Learning Machine (ELM). ELM has strong point in processing speed and suitable as real time classifier.

ELM, firstly introduced by Huang [1], [9], has some important concepts:

1) ELM is a supervised learning classifier using a matrix based on the target and the correspondence input from training data.

2) ELM network architecture uses single Hidden Layer Feedforward Network (SLFN) on figure 1. There is no known a priori standard to determine the exact number of hidden nodes (L). Generally, according to 


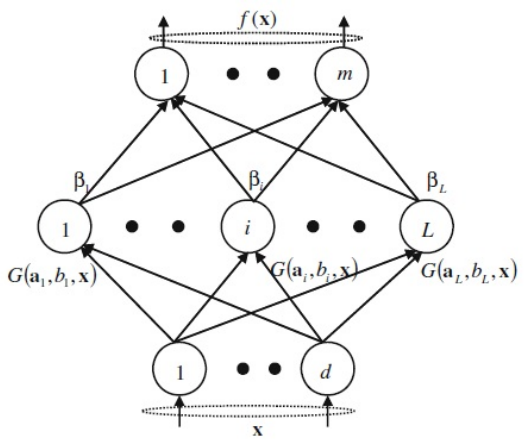

Fig. 1. Single-hidden layer feedforward network (SLFN)

Huang [2] from the interpolation theorem, L may vary depends but not larger than the training samples $(\mathrm{N})$. Larger $\mathrm{L}$ requires more computation and increases processing time. However, based on some previous experiments, better accuracy obtained by adding the number of hidden nodes.

3) ELM activation function on computing nodes can be any non linear infinitely differentiable function. From interpolation theorem [2], there is a wide type of activation functions can be used, which include the sigmoid functions, the radial basis, sine, cosine, exponential. Interestingly, according to the universal approximation theorem, the activation function can be extended to almost any type of nonlinear piecewise continuous function including the threshold function, hard Limit, triangular basis function, radial basis function (RBF) or even fuzzy activation function.

4) The weight input $w_{i}$ and bias $b_{i}$ are assigned with random numbers matrix sized by Number of L Hidden Neurons. According to the universal approximation theorem that SLFNs with randomly generated additive nodes can universally approximate any continuous target functions.

5) Matrix $\mathbf{H}$ is calculated as activation function $G$ of the summation matrix result from Bias Matrix $b_{i}$ and weight input $w_{i} \cdot x_{n}$ of $\mathrm{N}$ training data and $\mathrm{L}$ hidden nodes. Matrix $\mathbf{H}$ has still no information from learning process yet. Hidden layer matrix $\mathbf{H}$ is like an information base of training data without a knowledge.

$$
\mathbf{H}=\left[\begin{array}{ccc}
g\left(w_{1} \cdot x_{1}+b_{1}\right) & \cdots & g\left(w_{L} \cdot x_{1}+b_{L}\right) \\
\vdots & \ddots & \vdots \\
g\left(w_{1} \cdot x_{N}+b_{1}\right) & \cdots & g\left(w_{L} \cdot x_{N}+b_{L}\right)
\end{array}\right]
$$

6) Hidden node parameters now remain fixed after randomly generated. The ELM training is simply equivalent to finding a least-squares solution $\hat{\beta}$ of the linear system $\mathbf{H} \beta=\mathbf{T}$. The smallest norm least-squares solution is the Output weight $\hat{\beta}=\mathbf{H}^{\dagger} \mathbf{T}$, which $\mathbf{H}^{\dagger}$ known as Moore-Penrose generalized inverse (Pseudo Inverse) of $\mathbf{H}$. Output Weight is like a knowledge base from supervised learning driven by matrix $\mathbf{T}$ (Target output). In linear algebra, a matrix $\mathrm{A}$ has a unique inverse $A^{-1}$ only if it is square, and even then only if it is nonsingular. Generalized inverse actually is an approximation method to find the inverse of any general matrix which could be not unique solution. Different methods to calculate the Moore-Penrose generalized inverse of a matrix [4]: orthogonal projection method, orthogonalization method, iterative method, and singular value decomposition (SVD). The simplest method is SVD method, like pinv function in matlab. The orthogonal projection method can be efficiently used in ELM [2] with the ridge regression theory, a positive value $1 / \lambda$ is added to the diagonal of $\mathbf{H}^{T} \mathbf{H}$ or $\mathbf{H H}^{T}$, the result is stabler and tends to have better generalization performance. The simplified formula $\beta$ is :

$$
\beta=\left(\frac{\mathbf{I}}{\lambda}+\mathbf{H}^{T} \mathbf{H}\right)^{-1} \mathbf{H}^{T} \mathbf{T}
$$

During the testing, ELM will call the Output Weight $\beta$ and multiplied it with the information base obtained from input testing data to get the testing result. Although ELM has simplified, two major issues still remain in ELM.

1) The only factor that needs to be set by users is the structure size of the number of hidden nodes. How to choose the optimal number of hidden nodes is still unknown and users usually have to do trial-and-error.

2) Whether the computation complexity of ELM can be further reduced especially when given large number of training data and when large number of hidden nodes required.

Some papers explained how ELM algorithm used in human action recognition but not in pose-based recognition. Minhas [9] has introduced Incremental ELM in human action recognition based on snippets of shape of human actor by approximately changing intensity histograms to extract pyramid histograms of oriented gradient features. Venkatesh [11] has introduced Fully complex-valued ELM classifiers for human action recognition using the fully complex-valued hyperbolic secant as an activation function. Optical flow-based features extracted from the video sequences are utilized to recognize 10 different human actions. Nizami [6] has presented a multiview gait recognition algorithm for identification at a distance used two well known and effective gait representations namely Motion Silhouette Image (MSI) and gait energy image (GEI). The features for MSI and GEI images are extracted using Independent Component Analysis (ICA). Extreme Learning Machine (ELM) classifier is then used for classification. ELM is focused as fast classifier and the performance is increased simply by tuning the network parameter using hidden nodes number, training methods or activation function improvement.

Some papers in multi classification study with non ELM classifier, however, emphasize the importance of feature selection to reduce the dimension of data. The objective is to reduce the complexity and computation time of non ELM which significantly different with ELM classifier. One of the feature selection method is known as bag of words or bag of visual words (computer vision) or bag of correlated poses [1] and various ensemble techniques like boosting and bagging. 


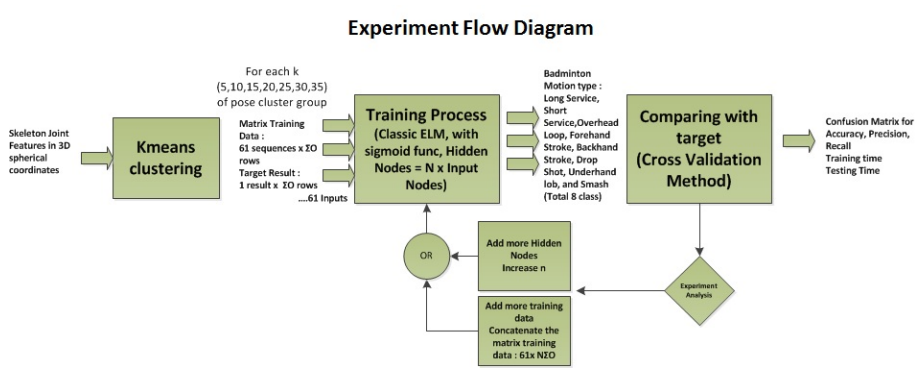

Fig. 2. Training Block Diagram

\section{METHODS}

The observation data of human gestures are captured using a static mounted Kinect depth camera positioned in front of the performer at the rate of $30 \mathrm{fps}$ with distance at 2-3 meters in closed area. It is working based on pattern grid reflection of near-to-infrared light emitter to the object [7]. Using openNI framework, kinect can generate skeleton information from human pose instantly in 3D coordinate $(x, y, z)$. Modifying Raptis [8] and Heryadi [13], skeleton joint features are represented by parameter of spherical 3D coordinate $\theta$ as inclination and $\varphi$ as azimuth. We used all joints except NECK (Small variance) with total $14 \theta$ and $14 \varphi$ multiplied by $\mathrm{N}$ frame sequences (Different for each motion). We used some basic motion from Badminton sport action and traditional dance (Balinese dance) to define the motion classes. The motion class for Badminton are Long Service, Short Service, Overhead Loop, Forehand Stroke, Backhand Stroke, Drop Shot, Underhand lob, and Smash (Total 8 class). Total training data is 235 rows and the longest sequence is 61 sequences. The motion class for traditional dance are Agem kanan, Agem kiri, Piles, Ngeseh, Luk Nerudut, Malpal, Ngegol, Mungkahlawang, and Nayog (Total 11 class). Total training data is 705 rows and the longest sequence is 451 sequences. We used Kmeans algorithm to cluster $28 \times \Sigma \mathrm{N}$ features data observation into a group of key poses. Each motion is represented as a sequence of cluster pose labels as string representation then converted using arithmetic series within range -1 to 1 because it is not a monotonic function. For validation, we used cross validation with Kfold $\mathrm{N}=5$.

\section{EXPERIMENT AND EVALUATIONS}

Comparison with another statistical classifier Nearest Neighbor family (K-NN, Fuzzy K-NN and CNN), ELM is the fast in processing time even with the big size of network structure to approach the same generalization accuracy level (In Table I, ELM3 with 61 inputs, 30x61 Hidden layer Nodes and concatenated $30 \times 235$ training rows has testing time $0.12 \mathrm{~s}$ for 60 testing rows; K-NN required 4.1s, Fuzzy K-NN required $7.16 \mathrm{~s}$ and $\mathrm{CNN}$ required $0.62 \mathrm{~s}$ per testing row). However, the learning method by increasing the network structure to improve the performance not always reliable and not giving the best solution all the time.

\section{A. Increasing The Network Structure}

We increased the $\mathrm{L}$ hidden nodes number by $\mathrm{n} x$ input sequence number, until larger than training data rows. In figure 3 , Badminton motion, the increase of hidden nodes is not
TABLE I. ACCURACy Percentage for all Class in Badminton MOTION.

\begin{tabular}{|c|c|c|c|c|c|c|}
\hline Pose Cluster & K-NN & FK-NN & C-NN & ELM1 & ELM2 & ELM3 \\
\hline \hline 5 & 97.96 & 98.994 & 89.145 & 60.111 & 64.203 & 68.045 \\
\hline 15 & 88.125 & 98.327 & 89.506 & 53.896 & 54.497 & 95.831 \\
\hline 25 & 79.777 & 98.293 & 88.829 & 35.244 & 44.073 & 97.797 \\
\hline
\end{tabular}

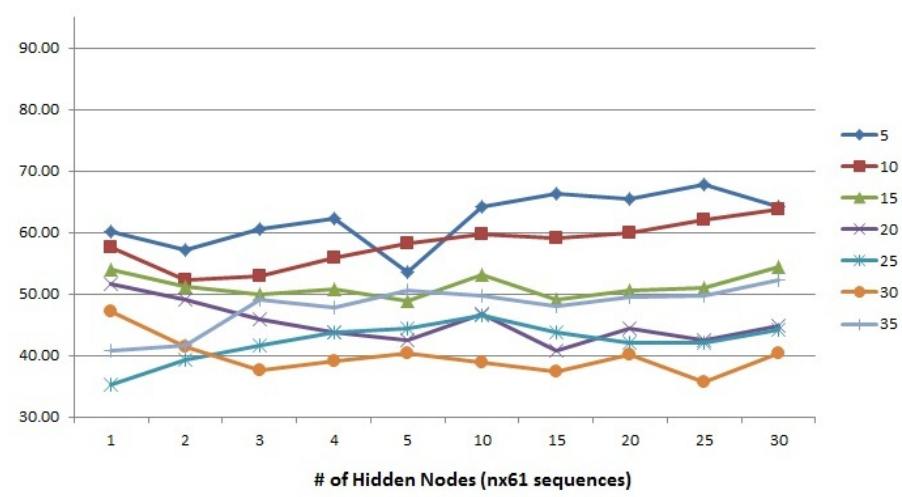

Fig. 3. Accuracy against \# of Hidden Nodes with 235 rows training data for Badminton motion (all classes)

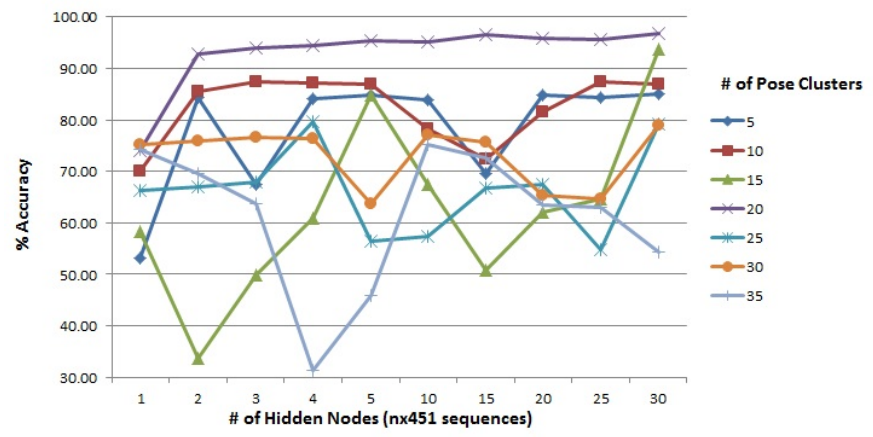

Fig. 4. Accuracy against \# of Hidden Nodes (nx451) with 705 rows training data for Dance motion (all classes)

giving any contribution significantly to the accuracy (Less than $10 \%$ fluctuated) unless adding more training data with good in big pose clusters only. In certain pose, adding more hidden nodes and training data, makes the accuracy fluctuated (unstable). In figure 4, dance motion which is more regular, the increase of hidden node numbers not giving the best accuracy and tends to be unstable with $20 \%$ variance except for pose cluster=20. Bigger pose clusters tend to have lower accuracy performance. It seems for both regularity and no regularity data, the network has a congestion limit. Increasing the hidden nodes number and training data, not even giving contribution significantly to the performance. This is related with the interpolation theorem based on Huang explanation even $L \leq N$ actually is not the hard limit.

\section{B. Selecting The Training Data}

Another method is by training data selection to get only qualified training data into learning process. We select the training data with intra class variance $\geq 0.25$ and with inter class p-values for testing the hypothesis of no correlation with value $0.75 \leq \mathrm{p}<1$. The overall accuracy is decreased 


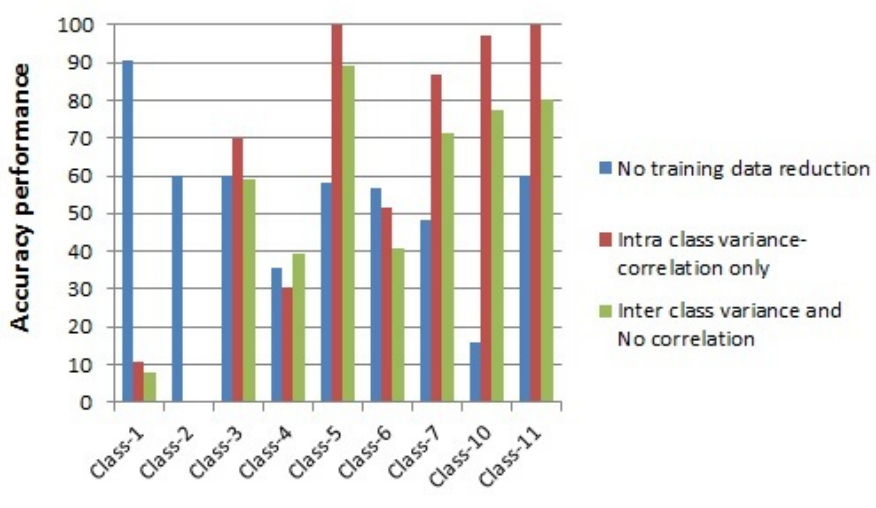

Fig. 5. Accuracy performance of Dance motion per class with full and selected training data
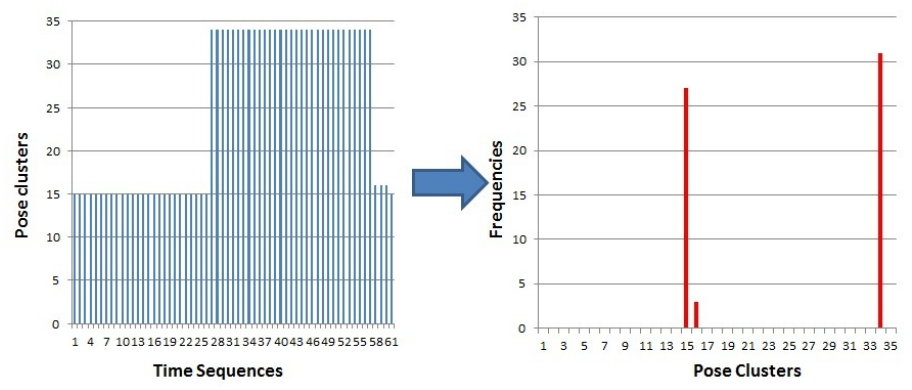

Fig. 6. Bag of Pose: the pose frequency of occurence

because certain class has no training data left at all, thus the accuracy is 0 . In another class, no data filtered at all, thus the accuracy remains the same. For certain class in dance motion, the performance is improved by reducing data similarity. But not adequate training data, will make the generalization performance is not well performed (Figure 5). However, this method is impractical because it is not easy to find the optimum statistic parameter for uncertain type of data.

\section{Feature Selection}

This paper proposes to use the frequency of pose occurrences as features rather than time sequence of pose. Similar to bag of words, we called it bag of pose. It significantly reduces the dimension of training data because pose sequence in time domain actually has sequence location and the occurrence frequency. The frequency of pose occurrence has more distinction between the class, so the accuracy can be improved.

Both badminton and dance motion using bag of pose showed improved accuracy using single ELM classifier with inverse function 0 ( $\mathrm{svd} / \mathrm{matlab}$ pinv) on figure 7 compared with fig. 3 and figure 8 compared with fig. 4. Alternatively, with inverse function 1 using Equation 2 has stabler accuracy (15$20 \%$ higher) on figure 9 compared with fig. 10 and figure 11 compared with fig. 12.

The appropriate feature selection is very important and has to be decided in the beginning. Refer to table II, using the bag of pose has significant impact to the reduced hidden nodes number and stabler inverse function selection to get better

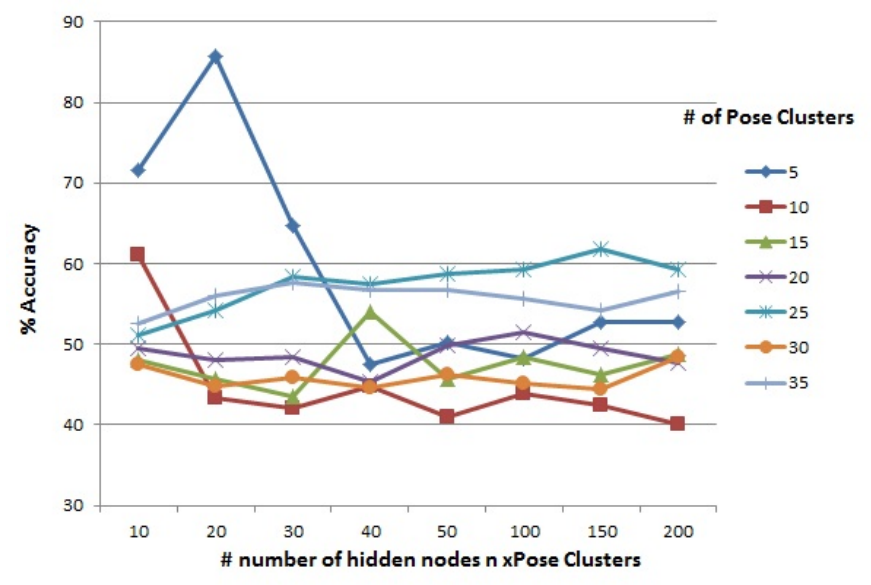

Fig. 7. Accuracy of badminton motion recognition (all classes) in bag of pose (all)

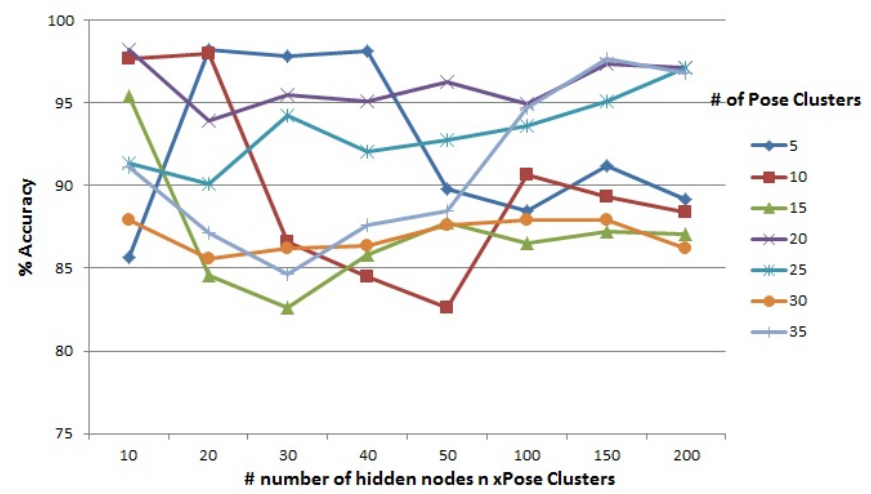

Fig. 8. Accuracy of dance motion recognition (all classes) in bag of pose (all)

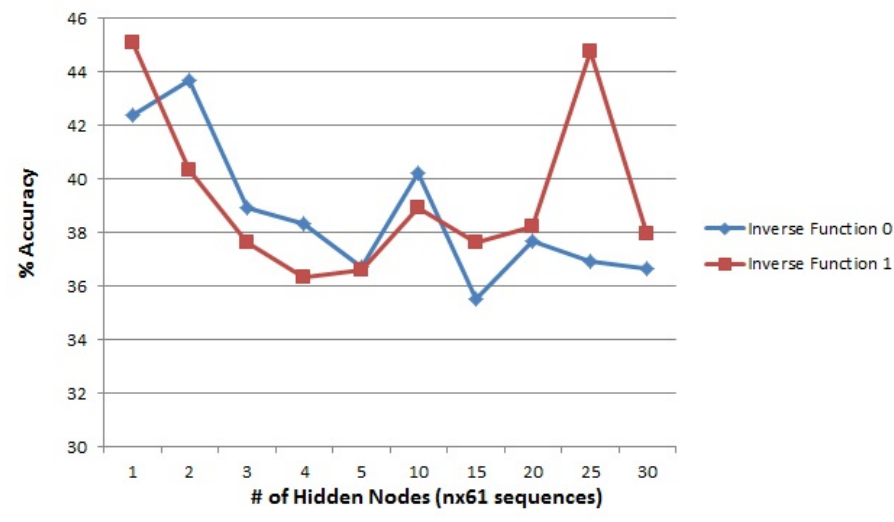

Fig. 9. Accuracy of badminton motion recognition (all classes) in time sequences $($ pose $=35)$

performance in accuracy as well as faster training processing time.

\section{CONCLUSIONS AND DisCUSSIONS}

The way to improve ELM classifier generalization accuracy performance can be obtained by selecting the feature of training data from more complex dimension size in time sequence 


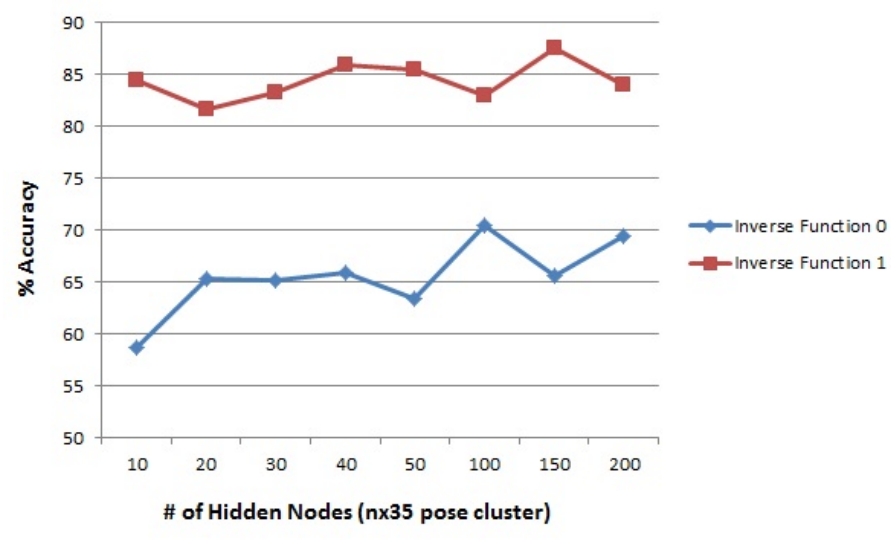

Fig. 10. Accuracy of badminton motion recognition (all classes) in bag of pose $($ pose $=35)$

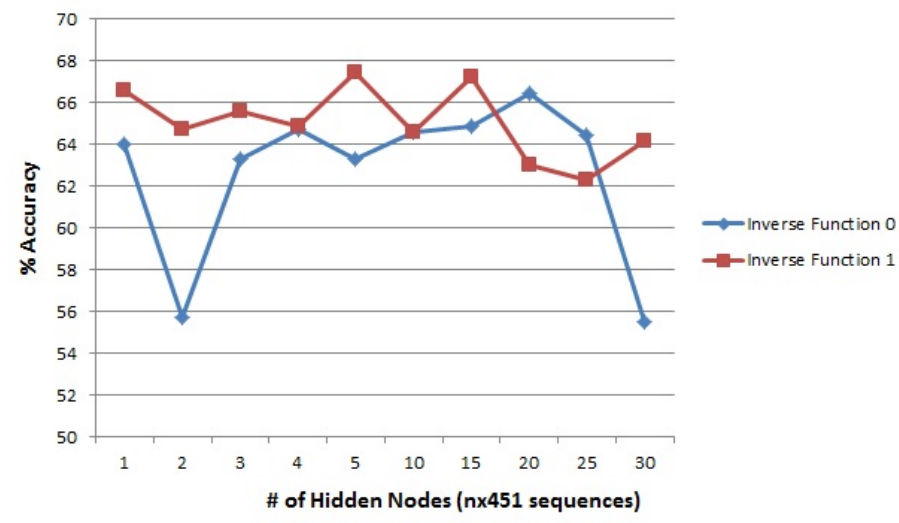

Fig. 11. Accuracy of Dance motion recognition (all classes) in time sequences (pose $=35)$

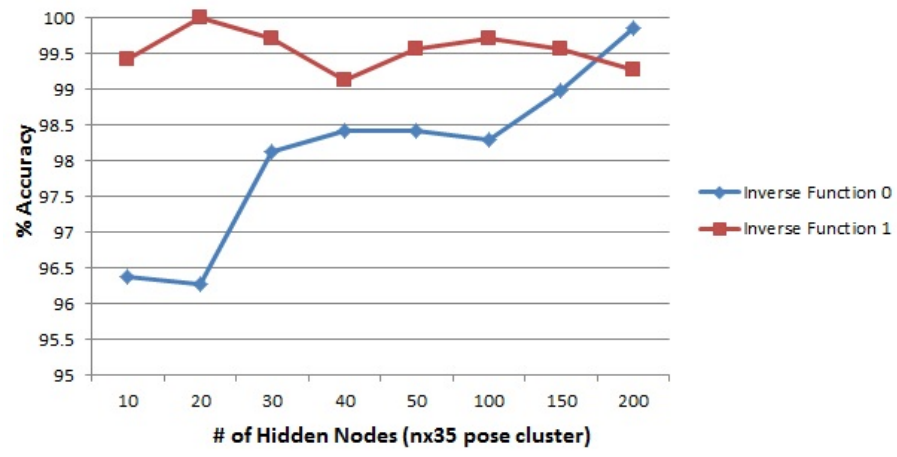

Fig. 12. Accuracy of Dance motion recognition (all classes) in bag of pose (pose $=35)$

to be more simple and distinguished in frequency of occurrence (bag of poses). Addressing accuracy performance on ELM, we can improve without adding network structure complexity by selecting the optimum feature of training data first. So, we can make sure the ELM performance well improved with just simple network structure. We evaluate its effectiveness in classifying motions with different degree of regularity (freedom). Eventhough statistically, the frequency feature selection might be better as the selected feature, but syntactic the pose location
TABLE II. ELM PERformance - Badminton Class ACCURACY IN $\%$ (Pose CLUSTER=35)

\begin{tabular}{|l|c|c|c|c|}
\hline Time-30x61 Seq (Training time) & Class 1 & Class 2 & Class 3 & Class 4 \\
\hline \hline Inv function 0 (3.0576s) & 17.5 & 87.143 & 16.429 & 63.929 \\
\hline Inv function 1 (2.6832s) & 55 & 86.786 & 22.5 & 38.571 \\
\hline \hline Time-30x61 Seq (Training time) & Class 5 & Class 6 & Class 7 & Class 8 \\
\hline Inv function 0 (3.0576s) & 13.929 & 68.571 & 20.357 & 0 \\
\hline Inv function 1 (2.6832s) & 23.929 & 42.143 & 26.786 & 0 \\
\hline \hline Freq-20x35 Pose (Training time) & Class 1 & Class 2 & Class 3 & Class 4 \\
\hline \hline Inv function 0 (1.2324s) & 82.5 & 86.429 & 50 & 60.714 \\
\hline Inv function 1 (0.6084s) & 92.5 & 97.5 & 73.571 & 88.571 \\
\hline \hline Freq-20x35 Pose (Training time) & Class 5 & Class 6 & Class 7 & Class 8 \\
\hline Inv function 0 (1.2324s) & 71.071 & 81.786 & 41.071 & 41.429 \\
\hline Inv function 1 (0.6084s) & 72.143 & 92.143 & 78.929 & 51.905 \\
\hline
\end{tabular}

probably still has important meaning to be studied further in our future works.

\section{ACKNOWLEDGMENT}

The Ministry of Research and Technology, Republic of Indonesia, funded this work under the National Synergy Research Grant SINas, contract No. RT-2012-1117

\section{REFERENCES}

[1] Di Wu, and Ling Shao, "Silhouette Analysis-Based Action Recognition Via Exploiting Human Poses," Circuits and Systems for Video Technology, IEEE Transactions on, vol.23, no.2, pp.236,243, Feb. 2013.

[2] G.-B. Huang, D. H. Wang, and Y. Lan, "Extreme learning machines: a survey," International Journal of Machine Learning and Cybernetics, vol. 2, no. 2, pp. 107-122, 2011.

[3] G.-B. Huang, Q.-Y. Zhu, and C.-K. Siew, "Extreme learning machine: Theory and applications," Neurocomputing, vol. 70, pp. 489-501, 2006.

[4] G.-Bin Huang, H. Zhou, X. Ding, and R. Zhang, "Extreme Learning Machine for Regression and Multiclass Classification," IEEE Trans. On Systems, Man, and Cybernetics, vol. 42, no. 2, April 2012.

[5] H.-J. Rong, G.-B. Huang, and Y.-S. Ong, "Extreme learning machine for multi- categories classification applications," Neural Networks, 2008. IJCNN 2008, pp. 1709-1713, 2008.

[6] I. Nizami, S. Hong, H. Lee, S. Ahn, K.-A. Toh, and E. Kim, "Multiview gait recognition fusion methodology," Industrial Electronics and Applications, 2008. ICIEA 2008. 3rd IEEE Conference, pp. 2101-2105, 2008.

[7] K. Khoshelham and S. O. Elberink, "Accuracy and resolution of kinect depth data for indoor mapping applications," Sensors, vol. 12, pp. 14371454, 2012.

[8] M. Raptis, D. Kirovskiče, and H. Hoppe, "Real-time classification of dance gestures from skeleton animation," ACM SIGGRAPH Symposium on Computer Animation, 2011.

[9] R. Minhas, A. Mohammed, and Q. Wu, "Incremental learning in human action recognition based on snippets," Circuits and Systems for Video Technology, IEEE Transactions, vol. 22, no. 11, pp. 1529-1541, 2012.

[10] R. Poppe, "A survey on vision-based human action recognition," $E L$ SEVIER Image and Vision Computing, vol. 28, pp. 976-990, 2010.

[11] R. Venkatesh Babu and S. Suresh, "Fully complex-valued elm classifiers for human action recognition," Neural Networks (IJCNN), pp. 2803-2808, 2011.

[12] W. H. Liang Wang and T. Tan, "Recent development in human motion analysis," ELSEVIER Pattern Recognition, vol. 36, pp. 585-601, 2003.

[13] Y. Heryadi, M. Fanany, and A. Arymurthy, "A syntactical modeling and classification for performance evaluation of bali traditional dance," Advanced Computer Science and Information Systems (ICACSIS), pp. 261-265, 2012 